Esta revista forma parte del acervo de la Biblioteca Jurídica Virtual del Instituto de Investigaciones Jurídicas de la UNAM

\title{
MARGARET MARTIN*
}

1. ¿Qué es la filosofía del derecho? ¿Cuál es su metodología adecuada?

\section{Método}

La aproximación metodológica que es apropiada para la investigación jurisprudencial es un método focal o basado en ideales mediante el cual uno explora las diferencias entre sistemas jurídicos ideales y sus contrapartes menos deseables. Para entender por qué este método es valioso, es útil considerar algunos de los defectos graves de la metodología dominante antes de volver a las que considero son las virtudes de una aproximación basada en ideales. El método de casos centrales es a menudo subestimado como una investigación normativa, una asignatura inadecuada para la investigación en la filosofía del derecho.

Este paso es muy apresurado, por las siguientes razones. Considere la idea de que lo que el derecho es puede ser distinguido de lo que debe ser. ${ }^{1}$ Para ver los límites de este credo positivista, es útil considerar por qué este eslogan es tan poderoso: siempre hay una perspectiva desde la cual una norma dada puede ser evaluada. Cierta norma jurídica o cierto sistema de normas siempre pueden ser sometidas a una crítica moral. Así que la segunda mitad del eslogan es verdadera, casi que por definición. Es la primera mitad del credo la que desafía de forma seria a los positivistas $¿$ Es el derecho sólo una cuestión de hechos? Más específicamente, ¿se entiende mejor al derecho como un conjunto fáctico de normas? ¿Puede el con-

* University of Western Ontario. Faculty of Law.mmart2@uwo.ca

1 Para determinar si el derecho es fundamentalmente una herramienta, debemos precisar si es igualmente útil para el bien y para el mal. Este debate nos lleva más allá de las observaciones básicas mencionadas arriba. Véase el debate entre Simmonds, N. E. y Kramer, Matthew. El más reciente intercambio puede ser encontrado en el American Journal of Legal Theory. Véase Kramer, M. H., "Incentives, Interests, and Inclinations: Legal Positivism Redefended", American Journal of Jurisprudence, vol. 51, 2006, pp. 165-178; Simmonds, N. E., "Evil Contingencies and the Rule of Law: a Response to Hamish Stewart", American Journal of Jurisprudence, vol. 51, 2006, pp. 179-190. 
Esta revista forma parte del acervo de la Biblioteca Jurídica Virtual del Instituto de Investigaciones Jurídicas de la UNAM

cepto de los sistemas jurídicos ser simplemente descrito?, o ¿entran los juicios de valor implícita o explícitamente al hacer tales determinaciones? Estas preguntas son más difíciles de responder afirmativamente de lo que uno podría asumir.2 También podemos preguntar si el método habitual de los positivistas - buscar el denominador común entre los sistemas jurídicos existentes - contribuye a iluminar la naturaleza del derecho.

Se pueden plantear serias dudas acerca del valor de este método una vez que resulte claro qué tipos de preguntas quedan excluidas. No podemos, por ejemplo, preguntar sobre las habilidades del derecho para suscitar el orden y permanecer fieles al método hartiano (como se esbozó arriba). La preocupación no es simplemente porque las suposiciones implícitas de valor acompañan a la explicación funcional; ${ }^{3}$ el miedo también es a que al centrarse en la función del derecho no se puedan producir resultados que sean "universales" en el sentido deseado del término (i. e. compartido entre todos los sistemas existentes). ${ }^{4}$ El problema es que si examinamos los sistemas jurídicos existentes, pronto descubrimos que hay más de una forma en la que el derecho puede contribuir al orden social. El miedo es el mecanismo central en algunos regímenes, en tanto que la aceptación internalizada juega un papel fundamental en ayudar al derecho a suscitar el orden.

Los filósofos del derecho se enfrentan a una encrucijada. Ellos pueden explorar las diferentes formas en las que el derecho puede contribuir al orden, o ellos pueden decidir simplemente no hacer esta pregunta. Yo abogo por la segunda de las dos opciones, en tanto que muchos positivistas destacados siguen el primer camino. Dado que la relación del derecho con el orden es central a nuestra experiencia de derecho, parece lógico tratar de entender cómo el derecho contribuye al orden, aun si esto significa que debamos enfocarnos en las diferencias entre los sistemas jurídicos, en lugar de sus características comunes.

2 Martin, M., Judging Positivism, Oxford, Hart Publishing, 2014.

3 Esto también es una preocupación, véase Martin, Judging Positivism, cit.

${ }^{4}$ Green, L., "Law as a Means", en Cane, P. (ed.), The Hart-Fuller Debate in the Twenty-First Century, Oxford, Hart Publishing, 2010, p. 84. 
Esta revista forma parte del acervo de la Biblioteca Jurídica Virtual del Instituto de Investigaciones Jurídicas de la UNAM

Es importante tener presente que la pregunta sobre el rol del derecho para suscitar el orden fue considerada fundamental por positivistas prominentes, quienes ahora rehúyen tales explicaciones. ${ }^{5}$ El método positivista insta a los filósofos del derecho a detener sus investigaciones cada vez que la apreciada división entre hechos y valores es violada, o cada vez que el denominador común no puede encontrarse. ${ }^{6}$ No es un "hecho" que el método conceptual sea el camino para la verdad en la jurisprudencia. La filosofía, a diferencia de otras disciplinas, siempre considera a la verdad como un asunto a tratar.

Para entender por qué el método de los casos centrales no puede ser subestimado como una investigación puramente normativa, hay que ver que el producto de este método es incompatible con el producto de la aproximación hartiana y también le compite. El ejemplo al que siempre vuelvo para ilustrar este aspecto es uno que propone Aristóteles, célebre por utilizar el método de los casos centrales. ${ }^{7}$ Para entender la naturaleza de la amistad, Aristóteles sostiene que lo que debemos comprender es su manifestación ideal. El caso ideal de amistad es el de dos personas que se desean a cada uno el bien por razón del otro. ${ }^{8}$ Hay otros tipos de amistad que no alcanzan el nivel del ideal. Algunas amistades se basan en el placer y cesan cuando el placer cesa; otras en la utilidad. Una vez más, cuando la persona no es útil, la amistad desaparece. Estas dos formas de amistad son transitorias - la otra persona es sólo un medio para los propios fines egoístas- El caso ideal es la única versión desinteresada de la amistad, y más estable y más deseable. El universal es entendido a través de los lentes del caso ideal.

5 Compare los siguientes dos pasajes: Raz, J., The Authority of Law: Essays on Law and Morality, New York, Oxford, Oxford University Press, 1979, pp. 50 y 51, y Raz, J., "Postema on Law's Autonomy and Public Practical Reason: a Critical Commentary", Legal Theory, vol. 4, núm. 1, 1999, pp. 3-11.

6 En realidad, es muy difícil mantener separados a los dos reinos. Véase Martin, Judging Positivism, cit.

7 Martin, M., "Raz's The Morality of Freedom: Two Models of Authority”, Jurisprudence, vol. 1, núm. 1, 2010, p. 64.

8 Aristóteles, Ética a Nicómaco, 11598b 10 - 1159a 15. 
Esta revista forma parte del acervo de la Biblioteca Jurídica Virtual del Instituto de Investigaciones Jurídicas de la UNAM

Al comparar las similitudes y diferencias entre los casos centrales y las contrapartes disfuncionales, podemos ganar un entendimiento más claro de los tres tipos de amistades. No estamos simplemente interesados en cómo debe ser la amistad, sino más bien en entender las relaciones como se dan en este mundo. El método del caso central compite con el método hartiano. Si aplicamos el método hartiano al concepto de amistad, descubrimos que se suprimen las características más importantes que emergen del análisis aristotélico. Por supuesto, esto es deliberado. Cuando buscamos los puntos comunes entre todos los tipos de amistad, obtenemos, de forma predecible, un concepto pobre: los amigos se conocen, gastan el tiempo juntos y aparentemente se agradan entre sí. En realidad, no entendemos ningún tipo de amistad, aún más (diría yo), el concepto hartiano no nos ayuda a entender lo que la amistad es.

En resumen, es a través de la comparación detallada de los casos existentes de una especie lo que ilumina aquello sobre lo que deseamos explorar. En mi opinión, lo mismo es válido para el derecho: la construcción de un concepto pobre de derecho que emerge cuan-do buscamos los puntos comunes entre los sistemas jurídicos no es el camino hacia el entendimiento filosófico. Es una mera abstracción que nos dice extraordinariamente poco acerca del derecho tal como lo encontramos (y aún menos si nos adherimos estrictamente al método y dejamos a los sociólogos explorar la pregunta sobre la relación entre el derecho y el orden).

Una teoría del derecho de los casos centrales emerge del simple acto de comparar los sistemas existentes - por ejemplo, aquellos que son dominados por el miedo - y aquellos en los que la aceptación es la más destacada relación que los ciudadanos tienen con el derecho (un ideal también puede emerger por otros caminos que también son esclarecedores). ${ }^{9} \mathrm{Al}$ entender en mayor detalle cómo el derecho ordena a las sociedades, podemos entender más claramente las características que distinguen los buenos sistemas de sus contrapartes perversas.

9 Simmonds, N. E., Law as a Moral Idea, Oxford, Oxford University Press, 2007; Finnis, J., Natural Law and Natural Rights, Oxford, Oxford University Press, 2011. 
Esta revista forma parte del acervo de la Biblioteca Jurídica Virtual del Instituto de Investigaciones Jurídicas de la UNAM

MARGARET MARTIN

\section{Preguntas}

Las preguntas que son importantes y que deberían ser abordadas por los filósofos son numerosas. La naturaleza de las preguntas que pueden ser planteadas y las respuestas dadas no pueden ser predeterminadas. Una vez que la distinción normativa/descriptiva es revelada como solo una de las formas de pensar acerca del derecho (y una muy discutida), entonces el debate jurisprudencial puede ser expandido. En lugar de rechazar rápidamente aproximaciones alternativas como "no jurisprudenciales", los filósofos del derecho deberían sentirse exhortados a debatir con sus competidores en la búsqueda de ideas acerca de la naturaleza del derecho. Las preguntas sobre el método y la sustancia están siempre sobre la mesa, por así decirlo.

Una pregunta que me interesa (y que espero explorar en el futuro) es la forma en la que el derecho contribuye o impide traer justicia al mundo. Al ser ubicado en el contexto del método de los casos centrales, se hace posible ver las conexiones entre el derecho y la justicia que pueden ser inherentes al caso ideal. ${ }^{10}$ También es útil pensar la pregunta con relación al derecho internacional. Es una nueva frontera donde las ideas acerca del derecho (y la justicia) informan el trabajo de los abogados internacionalistas. Es importante pensar a través de los ideales jurídicos, porque su implementación afecta las vidas de muchos. ${ }^{11}$ La teoría y la práctica se entrecruzan.

2. ¿Cuál es su experiencia personal? ¿Cómo comenzó en la filosofía del derecho? ¿Qué personas le influenciaron sustancialmente en su trabajo?

Mi primer encuentro con la filosofía jurídica fue en un curso de posgrado enseñado por Wil Waluchow. Fue en excelente curso que cubrió

10 Nótese que podemos ver cómo la "tesis de la separación" sólo tiene sentido si uno adopta la metodología hartiana.

11 Martin, M., "Between Utopian Dreams and Political Realities", en TanquayRenaud, F. y Stribopoulos, J. (eds.), Rethinking Criminal Law Theory: New Canadian Perspectives in the Philosophy of Domestic, Transnational, and International Criminal Law, Oxford, Hart Publishing, 2012. 
Esta revista forma parte del acervo de la Biblioteca Jurídica Virtual del Instituto de Investigaciones Jurídicas de la UNAM

muchos de los debates contemporáneos. Fui expuesta a todos los gigantes en el campo - Hart, Dworkin, Raz, Coleman y Leiter (para nombrar sólo unos pocos)-. Me sentí particularmente atraída a la discusión sobre la relación entre método y sustancia - Brian Bix, Frederick Schauer y John Finnis fueron autores que plantearon preguntas sobre las que continúo reflexionando-. De hecho, mi trabajo principal se centra en dichos temas. Mi interés no es principalmente con la sustancia de las diferentes posiciones, sino también con la metodología. Como mencioné antes, me preocupa el estado del debate hoy en día, y en particular la tendencia a interrumpir prematuramente el diálogo entre las posiciones divergentes —una cuestión que Andrew Halpin articula muy convincentemente en un artículo reciente-. ${ }^{12}$

He sido muy influenciada por el trabajo de N. E. Simmonds. Me inspiraron sus primeros trabajos, una colección de reflexiones críticas sobre el statu quo de la filosofía del derecho. ${ }^{13}$ La belleza de su trabajo está en que plantea más preguntas de las que responde (y responde muchas). Los escritos de Lon Fuller tienen una característica similar. Fuller es menos sistemático en sus aproximaciones a la teoría jurídica que muchos de sus adversarios, pero él está constantemente reflexionando sobre varios aspectos de la práctica jurídica, moviéndose constantemente entre ideas abstractas y ejemplos particulares del mundo real, dejando un sendero de preguntas para que los futuros jurisprudentes reflexionen. The Law in Quest of Itself (El derecho en busca de sí mismo ${ }^{14}$ es uno de mis favoritos - es un gran libro con un título maravilloso-.

12 Halpin, A., "Austin's Methodology? His Bequest to Jurisprudence”, Cambridge Law Journal, vol. 70, 2011, pp. 175-202.

13 Véase, por ejemplo, Simmonds, N. E., "Bluntness and Bricolage”, en Gross, H. and Harrison, R. (ed.), Jurisprudence Cambridge Essays, Oxford, Clarendon Press, 1992; Simmonds, N. E., "Between Positivism and Idealism", Cambridge Law Journal, vol. 50, 1991, pp. 308-329.

14 Fuller, L., Law in Quest of Itself, Boston, Beacon Press, 1966. 
Esta revista forma parte del acervo de la Biblioteca Jurídica Virtual del Instituto de Investigaciones Jurídicas de la UNAM

3. ¿Cuáles son las áreas y temas en los que ha trabajado en filosofía jurídica? ¿Cuáles han sido sus mayores influencias?

Mi trabajo principal es una crítica del positivismo jurídico (especialmente a la versión de Raz). En Judging Positivism, ${ }^{15}$ demuestro la forma en la que Raz altera su teoría a lo largo del tiempo y cómo esas alteraciones han producido profundas inconsistencias e incoherencias en sus posiciones. Mi objetivo central es resaltar cómo muchas de las suposiciones establecidas que hoy son tratadas como verdades son discutibles y necesitan de una defensa. Yo exploro la distinción fundacional entre la jurisprudencia normativa y descriptiva, así como otras importantes distinciones (tales como la distinción entre una teoría jurídica y una teoría de la adjudicación) que a menudo estructuran el debate en la filosofía del derecho. Mi esperanza es que el debate pueda ser dinamizado. Si no hay un único camino al entendimiento iusfilosófico, entonces puede ocurrir una más amplia conversación.

También estoy interesada en explorar la naturaleza del derecho internacional con el fin de arrojar luz tanto al derecho doméstico como al internacional. Lo atractivo del derecho internacional es que las ideas realmente funcionan en la práctica, las ideas de los abogados internacionalistas acerca de lo que constituye el derecho y su capacidad para lograr la justicia. También me preocupo por las desventajas potenciales de las ambiciones nobles de los abogados internacionalistas. ${ }^{16}$ Creo que explorar las suposiciones en las que se basa el continuo desarrollo del derecho internacional es un importante proyecto en el que los teóricos del derecho pueden desempeñar un papel.

16 Martin, "Between Utopian Dreams and Political Realities", cit. 